\title{
Modelo experimental de un enfriador de aceite industrial
}

\section{Experimental model of an industrial oil cooler}

\author{
J. G. Hortelano-Capetillo@a,*, E. Baños-LópezĐb, J. L. Zuñiga-Cerroblanco@a \\ J. M. Martínez-Vázquez (iD) A. Pérez-Pérez \\ a Universidad Politécnica de Juventino Rosas, Miguel Hidalgo 102, Comunidad de Valencia, 38253, Juventino Rosas Guanajuato. \\ ${ }^{b}$ Escuela Superior de Tizayuca, Universidad Autónoma del Estado de Hidalgo, 43800, , Tizayuca-Hidalgo.
}

\begin{abstract}
Resumen
En el presente trabajo se diseña un modelo teórico que permite predecir el comportamiento térmico e hidráulico de un enfriador de aceite industrial tipo coraza y tubos, donde los fluidos de trabajo son aceite y agua. Se proponen los métodos $\varepsilon$-NTU, las correlaciones de Colburn, Gnieinski, Sieder Taste y Dittus Boelter para calcular los coeficientes convectivos internos y las correlaciones de Kern, Zukauskas, Hilpert y Churchill para los coeficientes convectivos externos. Se presenta la estructura mediante un software de manera numérica y gráfica, en función de los parámetros de operación y del diseño. El software permite modificar temperaturas, presiones de entrada y geometría de los equipos, obteniendo temperaturas de salida mediante la combinación de las correlaciones que se ajustan al proceso. Adicionalmente se realizaron pruebas experimentales y se compararon los resultados obtenidos con el modelo propuesto.
\end{abstract}

Palabras Clave:

Enfriador de aceite, fluidos de trabajo, comportamiento térmico, comportamiento hidráulico.

\begin{abstract}
In the present work a theoretical model is designed that allows predicting the thermal and hydraulic behaviour of an industrial oil cooler type shell and tubes, where the working fluids are oil and water. The $\varepsilon$-NTU methods, the Colburn correlations, Gnieinski, Sieder Taste and Dittus-Boelter to calculate the internal convective coefficients and the Kern correlations, Zukauskas, Hilpert and Churchill for the external convective coefficients. The structure is presented numerically and graphically, using software, depending on the operating and design parameters. The software allows modifying the inlet temperatures and pressures, the geometry of the devices, obtaining the outlet temperatures by combining of the correlations that fit the process. Additionally, experimental tests were carried out and the results were compared with the obtained according to the proposed model.
\end{abstract}

Keywords:

Oil cooler, working fluids, thermal behaviour, hydraulic behaviour.

\section{Introducción}

Un intercambiador de calor es un equipo que se utiliza para transferir calor entre 2 o más fluidos a diferentes temperaturas. Los intercambiadores de calor tipo coraza y tubos son equipos muy utilizados en la industria por su gran variedad de diseños y por la aplicación que tienen en los procesos de transferencia de calor, para la producción de energía convencional como condensadores, evaporadores, calentadores o enfriadores y regeneradores de vapor.

Los intercambiadores de calor más sencillos son los concéntricos, por el tubo interno circula un fluido, mientras que el otro fluido circula por el espacio anular. Dependiendo del sentido del flujo, se clasifica en flujo paralelo o contracorriente como se muestra en la Figura 1 (Kakac et al., 1998).

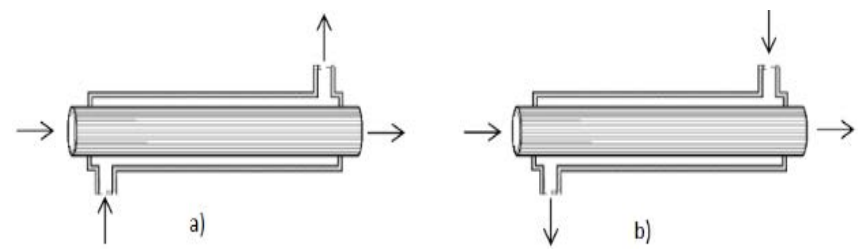

Figura 1: Intercambiadores de calor tubos concéntricos. a) Paralelo. b) Contracorriente.

Otra configuración común en intercambiadores de calor es el de carcasa y tubos. Las configuraciones difieren de acuerdo con el número de pasos por tubos y carcasa, la forma más simple implica un solo paso en la carcasa y tubos como se muestra en la Figura 2. En estos intercambiadores se instalan deflectores (bafles) para

\footnotetext{
*Autor para la correspondencia: jhortelano_ptc@upjr.edu.mx

Correo electrónico: jhortelano_ptc@upjr.edu.mx (J. Gregorio Hortelano Capetillo), esperanza_banoslo@hotmail.com (Esperanza Baños López), jzunigac_ptc@upjr.edu.mx (J. Luis Zuñiga Cerroblanco), dirección_ime@upjr.edu.mx (José Merced Martínez Vázquez), aperez_ptc@upjr.edu.mx (Arnulfo Pérez Pérez).
} 
aumentar el coeficiente de convección del fluido del lado de la carcasa (Kakac et al., 1998; Incropera et al., 1999).

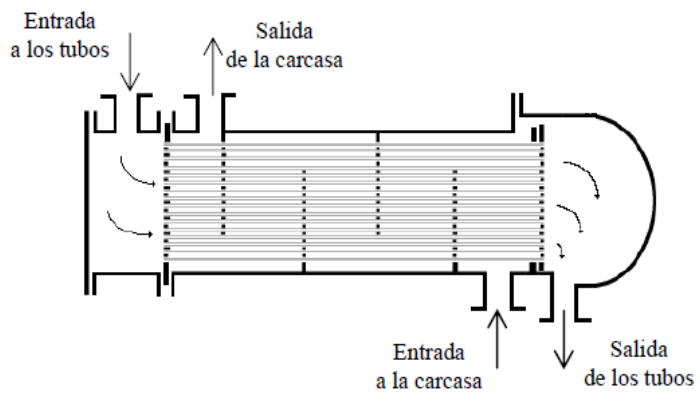

Figura 2: Intercambiador de calor de carcasa y tubos de un paso en la carcasa y tubos. (Flujo contracorriente).

En la Figura 3, se muestra la configuración de intercambiadores de calor tipo carcasa y tubos (Incropera et al., 1999).

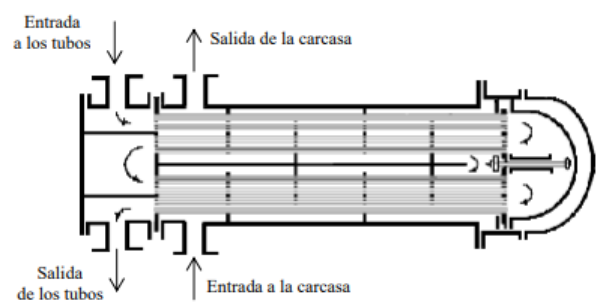

(a)

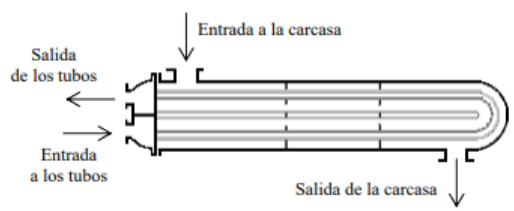

(b)

Figura 3: Intercambiadores de calor carcasa y tubos. a) Dos pasos por la carcasa y cuatro pasos por los tubos. b) Un paso por la carcasa y dos por los tubos.

Existen diversas publicaciones que proponen cambios en el diseño de intercambiadores, entre ellas la asociación Tubular Exchanger Manufacturers Association (TEMA). Sugieren tolerancias para la fabricación de componentes como espejos, bridas, tapas, boquillas, soportes, deflectores, tipo de materiales, entre otros (Gaddis et al., 2007). Diversos grupos de trabajo se han enfocado en modificar algunos componentes de los intercambiadores tipo carcasa y tubos.

Las correlaciones más empleadas para los diseños del lado de la carcasa son los métodos de Kern (Kern, 1973) y Bell Delaware (Bell, 1981). Existen investigaciones (Gay et al., 1982) enfocadas en la transferencia de calor, mientras que otros grupos (Gaddis et al., 1997) analizan las caídas de presión, otro factor que ha sido investigado es la transferencia de calor sobre los arreglos de los tubos (Karno et al., 2006). Por otra parte, también se han estudiado los efectos de la transferencia de calor y la caída de presión que causan el espaciamiento de baffles (Eryener et al., 2006).

Del mismo modo, surgen investigaciones mediante métodos computacionales, (Keene, 1994) tal es el caso de Keene, quien desarrollo un programa para validar experimentalmente un intercambiador de calor de carcasa y tubos, con visibles mejoras respecto a análisis realizado por sus antecesores, también incluyo el análisis de las caídas de presión entre las mamparas. Posteriormente Reppich y su Zagermann, con ayuda de un programa de optimización que utiliza el método de NewtonRaphson, así como el análisis de derivadas de segundo orden, generaron un modelo que optimiza costos en el diseño de los intercambiadores de calor tipo carcasa y tubos (Reppich et al., 1995). Por otra parte, Leong y colaboradores, diseñaron un software en Delphis Programming Languaje para el diseño de un intercambiador de calor carcasa y tubos, siendo los datos de entrada las condiciones de operación de los fluidos de entrada y salida; en el cálculo del coeficiente convectivo y la caída de presión del lado carcasa fue el método de Bell Delaware el utilizado, cabe mencionar que no presentan correlaciones para los coeficientes convectivos del lado de los tubos (Leong et al., 1998).

Poco después Su Thet Mon Than y colaboradores, diseñaron un software en Mathlab y Autocad para el diseño de un intercambiador de calor de carcasa y tubos, introduciendo los datos de entrada que son las condiciones de operación de los fluidos tanto del lado carcasa como lado tubos. Hicieron uso de correlaciones para el cálculo de coeficientes convectivos para flujo externo e interno, así como ecuaciones para los cálculos geométricos del intercambiador de calor y caídas de presión (Thet et al., 2008). En ese sentido el equipo de El-Fawal diseñaron un modelo para un intercambiador de calor donde calculan el área de transferencia de calor, caídas de presión, coeficientes convectivos para flujo externo e interno con correlaciones empíricas y algunos parámetros geométricos; además realizan un análisis para minimizar el área de transferencia de calor y obtener el menor costo del equipo (El-Fawal et al., 2011).

Trabajos posteriores de Kara y colaboradores presentan un modelo de intercambiadores de calor tipo carcasa y tubos mediante un código de programación realizado en Fortran 90, determinan la transferencia de calor óptimo respecto a las variables de entrada que corresponden con los flujos másicos, temperaturas de entrada y salida de los fluidos, en función del material empleado en los tubos (Kara et al., 2004). Dicho programa permite determinar las dimensiones generales del equipo, número de tubos, bafles, longitud, diámetro interno y externo de los tubos, área de superficie para la transferencia de calor, así como las caídas depresión.

Del mismo modo que Reppich diversos grupos continuaron realizando investigaciones para optimizar los costos en el diseño, fue así como Fettaka y colaboradores desarrollaron un método de diseño para intercambiadores de calor tipo carcasa y tubos (Fettaka et al., 2013).

Los trabajos mencionados, representan avances importantes, sin embargo, existe la necesidad de generar metodologías, que impulsen el desarrollo de herramientas para el diseño, fabricación y desempeño, así como cuantificar el rendimiento de enfriadores de aceite, para diseños de intercambiadores de calor.

Por lo anterior, en este trabajo se propone un modelo que permite simular y predecir el comportamiento térmico e hidráulico de un 
enfriador de aceite industrial tipo carcasa y tubos, para validar el modelo se realizaron mediciones experimentales.

\section{Desarrollo experimental}

En la Figura 4 se muestra el modelo experimental del enfriador de aceite, se emplearon termopares para conocer la temperatura de entrada y salida de ambos fluidos, con diferentes flujos volumétricos y temperaturas de entrada de agua para enfriar el aceite con flujo volumétrico y temperatura de entrada constante.

Los parámetros geométricos son: diámetro interno de la carcasa de $0.127 \mathrm{~m}$, longitud de los tubos $0.3048 \mathrm{~m}$, número de tubos 172 , pitch (espacio centro a centro entre tubos) $0.00794 \mathrm{~m}$, diámetro de los tubos internos $0.0055 \mathrm{~m}$, número de bafles 9 , número de pasos en los tubos 1, número de pasos en la carcasa 1 y arreglo triangular en los tubos.

El aceite caliente entra del lado tubos y el agua fría entra del lado carcasa. Los datos experimentales obtenidos fueron las temperaturas de entrada y salida para ambos fluidos medidos por termopares variando los flujos másicos mediante unos rotámetros.

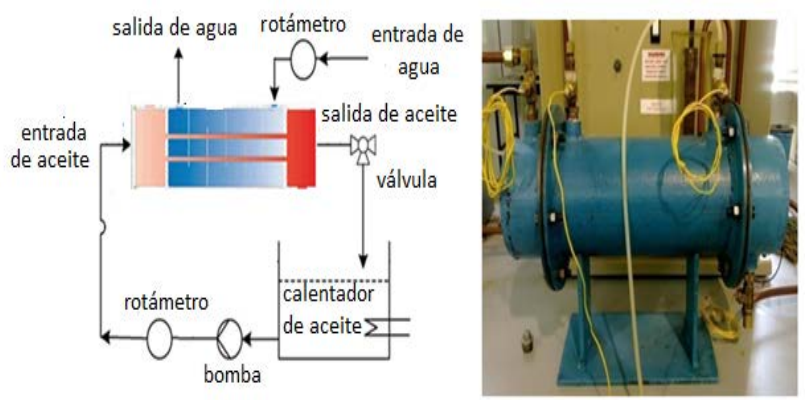

Figura 4: Diagrama esquemático de modelo experimental del enfriador de aceite.

Se analizaron 30 casos de estudio, los resultados obtenidos fueron: temperatura de entrada y salida de ambos fluidos, flujo másico del agua y gasto volumétrico del aceite, como se muestra en la Tabla 1. Del lado del aceite (Tubos) se modificaron los parámetros del gasto y la temperatura y del lado del agua (carcasa) se modificó el flujo másico a temperatura ambiente.

Tabla 1: Resultados experimentales obtenidos en el enfriador de aceite.

\begin{tabular}{ccccccc}
\hline & \multicolumn{3}{c}{ Lado Tubos (Aceite) } & \multicolumn{3}{c}{ Lado Carcasa (Agua) } \\
\hline $\begin{array}{c}\text { Número } \\
\text { de } \\
\text { Prueba }\end{array}$ & $\begin{array}{c}\text { Gasto } \\
(\mathrm{L} / \mathrm{s})\end{array}$ & $\begin{array}{c}\text { Temp. } \\
\text { Entrada } \\
\left({ }^{\circ} \mathrm{C}\right)\end{array}$ & $\begin{array}{c}\text { Temp. } \\
\text { Salida } \\
\left({ }^{\circ} \mathrm{C}\right)\end{array}$ & $\begin{array}{c}\text { Flujo } \\
\text { másico } \\
(\mathrm{g} / \mathrm{s})\end{array}$ & $\begin{array}{c}\text { Temp. } \\
\text { Entrada } \\
\left({ }^{\circ} \mathrm{C}\right)\end{array}$ & $\begin{array}{c}\text { Temp. } \\
\text { Salida } \\
\left({ }^{\circ} \mathrm{C}\right)\end{array}$ \\
\hline \hline 1 & 0.02 & 45 & 34.08 & 10.83 & 21.9 & 31.1 \\
2 & 0.02 & 44 & 32.42 & 10.83 & 21.92 & 30.5 \\
3 & 0.02 & 43 & 32.26 & 10.83 & 21.55 & 31.1 \\
4 & 0.02 & 42 & 31.04 & 10.83 & 20.9 & 29.32 \\
5 & 0.02 & 41 & 30.07 & 10.83 & 21.03 & 29.1 \\
6 & 0.02 & 40 & 30.58 & 10.83 & 21.05 & 29.37 \\
7 & 0.02 & 39 & 29.9 & 10.83 & 21.1 & 28.24 \\
8 & 0.02 & 38 & 30 & 10.83 & 21.12 & 28.51 \\
9 & 0.02 & 37 & 29.03 & 10.83 & 21.14 & 28
\end{tabular}

\begin{tabular}{ccccccc}
10 & 0.02 & 36 & 27.09 & 10.83 & 21.18 & 27.6 \\
11 & 0.02 & 35 & 27.65 & 10.83 & 21.11 & 26.5 \\
12 & 0.02 & 45 & 34.19 & 10.83 & 22.08 & 32.36 \\
3 & 0.019 & 45 & 35.07 & 10.83 & 22.1 & 32.59 \\
14 & 0.0181 & 45 & 34.02 & 10.83 & 22.13 & 32.83 \\
15 & 0.0171 & 45 & 33.22 & 10.83 & 22.13 & 33 \\
16 & 0.0162 & 45 & 33.48 & 10.83 & 22.18 & 33.3 \\
17 & 0.0152 & 45 & 33.08 & 10.83 & 22.28 & 33 \\
18 & 0.0143 & 45 & 33 & 10.83 & 22.28 & 29.9 \\
19 & 0.0133 & 45 & 32.78 & 10.83 & 22.37 & 29.5 \\
20 & 0.0123 & 45 & 32.6 & 10.83 & 22.34 & 31.2 \\
21 & 0.02 & 45 & 34.02 & 10.83 & 22.38 & 31.1 \\
22 & 0.02 & 45 & 34.03 & 10.5 & 22.41 & 31.5 \\
23 & 0.02 & 45 & 34.35 & 10.1 & 22.43 & 31.8 \\
24 & 0.02 & 45 & 34.46 & 9.8 & 22.46 & 32 \\
25 & 0.02 & 45 & 34.56 & 9.5 & 22.47 & 32 \\
26 & 0.02 & 45 & 34.82 & 9 & 22.61 & 32.1 \\
27 & 0.02 & 45 & 34.94 & 8.8 & 22.62 & 32.3 \\
28 & 0.02 & 45 & 35.14 & 8.5 & 22.68 & 32.7 \\
29 & 0.02 & 45 & 35.32 & 8.1 & 22.7 & 33 \\
30 & 0.02 & 45 & 35.42 & 7.8 & 22.73 & 33.6 \\
\hline \hline
\end{tabular}

\subsection{Descripción del modelo teórico.}

La propuesta o filosofía del modelo para el intercambiador de calor se presenta en la Figura 5. Se observa que los parámetros de entrada son: Datos geométricos del intercambiador de calor, flujos másicos, temperaturas y presiones de entrada de la carcasa y los tubos. En el modelo de simulación se determinan las propiedades térmicas de los fluidos y resuelve una serie de cálculos para después determinar las condiciones de operación de salida del intercambiador de calor carcasa y tubos como las temperaturas de salida, calor total, eficacia $(\varepsilon)$, número de unidades de transferencia de calor, por sus siglas en inglés (number of heat transfer unit) NTU, coeficiente global de transferencia de calor (U, $\left.\mathrm{W} / \mathrm{m}^{2}-\mathrm{K}\right)$ y los coeficientes convectivos internos $\left(\mathrm{h}_{\mathrm{i}}\right)$ y externos $\left(\mathrm{h}_{\mathrm{o}}\right)$. 


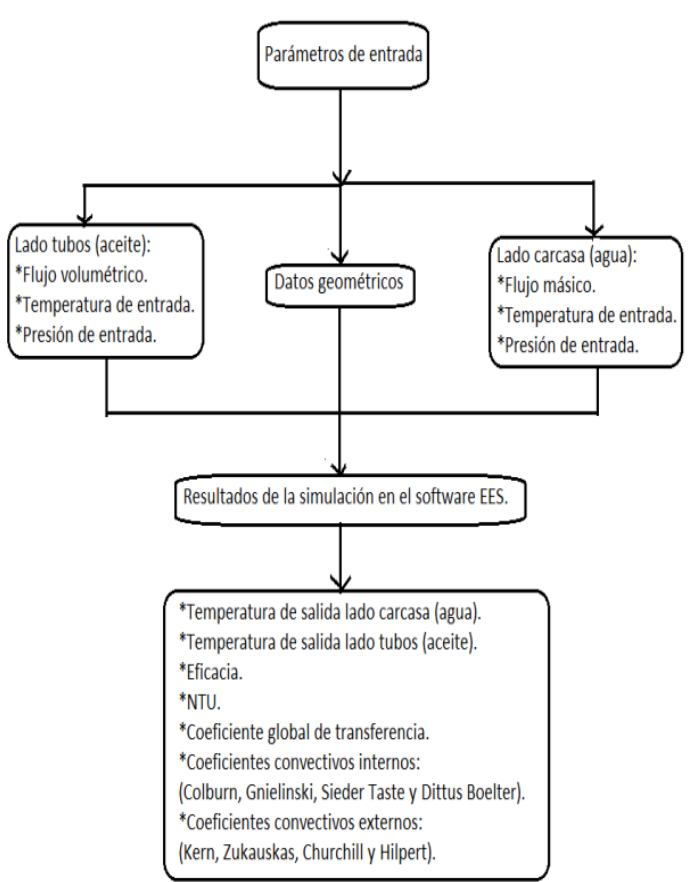

Figura 5: Diagrama de la estructura general del modelo propuesto (fuente propia).

El modelo teórico fue realizado en el software EES, por sus siglas en inglés (Engineering Equation Solver) usando los parámetros geométricos, el método $\varepsilon$-NTU y algunas correlaciones para calcular los coeficientes convectivos externos e internos. Las correlaciones para flujo externo son: Kern, Hilpert, Churchill y Zukauskas; para flujo interno son: Colburn, Gnielinski, Sieder Taste y Dittus Boelter.

A continuación, se muestra el método $\varepsilon-N T U$ (Kakac et al., 1998), representando el coeficiente global con los fenómenos de convección interna y externa, se muestra en la ecuación (1). Donde U (coeficiente global de transferencia de calor, $\mathrm{W} / \mathrm{m}^{2}-\mathrm{K}$ ), diámetro interno de los tubos $\left(\mathrm{D}_{\mathrm{int}}, \mathrm{m}\right)$, diámetro externo de los tubos $\left(D_{\text {ext }}, m\right), h_{i}$ (coeficiente convectivo interno, $\mathrm{W} / \mathrm{m}^{2}-\mathrm{K}$ ), $\mathrm{h}_{\mathrm{o}}$ (coeficiente convectivo externo, $\mathrm{W} / \mathrm{m}^{2}-\mathrm{K}$ ), $\mathrm{k}$ (conductividad térmica del material, $\mathrm{W} / \mathrm{m}-\mathrm{K}$ ):

$$
U=\frac{1}{\frac{D_{\text {ext }}}{D_{\text {int }} h_{i}}+\frac{D_{\text {ext }} \operatorname{Ln}\left(D_{\text {ext }} / D_{\text {int }}\right)}{2 k_{\text {mat }}}+\frac{1}{h_{o}}}
$$

El área total de transferencia de calor $\left(\mathrm{A}_{\text {total }}, \mathrm{m}^{2}\right)$, en función del diámetro interno de los tubos $\left(\mathrm{D}_{\mathrm{int}}, \mathrm{m}\right)$, longitud de los tubos $\left(\mathrm{L}_{\mathrm{t}}\right.$, m) y el número de tubos $\left(\mathrm{N}_{\text {tubos }}\right)$, se presenta en la ecuación (2):

$$
A_{\text {total }}=\pi D_{\text {int }} L_{t} N_{\text {tubos }}
$$

El análisis térmico se fundamenta en el método $\varepsilon$-NTU, la eficacia $(\varepsilon)$ y el NTU (número de unidades de transferencia de calor) se calcula como se presenta en ecuación (3), donde $\mathrm{C}_{\min }$ (capacitancia mínima, W/K) y $\mathrm{C}_{\max }$ (capacitancia máxima, $\mathrm{W} / \mathrm{K})$ :

$$
N T U=\frac{U A_{\text {total }}}{C_{\text {min }}}
$$

La efectividad se determina considerando el flujo contracorriente, como se presenta en ecuación (4), donde $\mathrm{Cr}$ (relación de capacitancias térmicas):

$$
\begin{aligned}
& \varepsilon=\frac{1-\exp [-N T U(1-C r)]}{1-C r \exp [-N T U(1-C r)]} \\
& C r=\frac{C_{\min }}{C_{\max }}
\end{aligned}
$$

Para calcular el calor máximo del intercambiador de calor $\left(\mathrm{Q}_{\max }\right.$, $W)$, se determina en función de la eficacia $(\varepsilon)$, la capacitancia mínima $\left(\mathrm{C}_{\text {min }}\right)$ y las temperaturas de entrada de los fluidos $\left(\mathrm{T}_{\text {ec }},{ }^{\circ} \mathrm{C}\right.$, temperatura de entrada carcasa, $\mathrm{T}_{\text {et }},{ }^{\circ} \mathrm{C}$, Temperatura de entrada tubos) como se expresa en la ecuación:

$$
Q_{\max }=\varepsilon \cdot C_{\min }\left(T_{e c}-T_{e t}\right)
$$

Una vez que se determina el calor total $\left(\mathrm{Q}_{\text {total }}, \mathrm{W}\right)$, se pueden estimar las temperaturas de salida de los fluidos lado carcasa y lado tubos mediante un balance de energía como se muestra en la ecuación (6), donde $\mathrm{m}_{\text {carc }}$ (flujo másico dentro de la carcasa, $\mathrm{kg} / \mathrm{s}$ ), $\mathrm{m}_{\text {tubos }}$ (flujo másico dentro de los tubos, $\mathrm{kg} / \mathrm{s}$ ), Cp carc (capacidad calorífica carcasa, J/kg-K), Cp tubo (capacidad calorífica tubos, $\mathrm{J} / \mathrm{kg}-\mathrm{K})$ :

$$
\begin{aligned}
& Q_{\text {total }}=m_{\text {carc }} C p_{\text {carc }}\left(T_{s c}-T_{e c}\right) \\
& Q_{\text {total }}=m_{\text {tubos }} C p_{\text {tubos }}\left(T_{e t}-T_{s t}\right)
\end{aligned}
$$

\subsubsection{Flujo Interno}

Existen un gran número de correlaciones en la literatura para fluidos turbulentos totalmente desarrollados sin cambio de fase en tubos circulares. El coeficiente convectivo de transferencia de calor se puede determinar con una correlación para el número de Nusselt en flujo turbulento totalmente desarrollado.

Correlación de Colburn se presenta en ecuación (7), con respecto a $\mathrm{Re}_{\mathrm{D}}$ (número de Reynolds, determina el flujo del fluido bajo condiciones laminar o turbulento) y Pr (número de Prandtl, determina el espesor relativo de las capas límites de momento y térmica), Fuente: (Colburn, 1933):

$$
N u_{D}=0.023 \operatorname{Re}_{D}^{4 / 5} \operatorname{Pr}^{1 / 3}
$$

Correlación de Gnielinski considera el factor de fricción (f), se presenta en ecuación (8), Fuente:(Gnielinski, 1976):

$$
N u_{D}=\frac{\left(\frac{f}{8}\right)\left(R e_{D}-1000\right) P r}{1+12.7\left(\frac{f}{8}\right)^{0.5}\left(\operatorname{Pr}^{0.66}-1\right)}
$$

Correlación de Sieder-Taste considera la viscosidad dinámica, se presenta en ecuación (9), Fuente: (Sieder et al., 1936):

$$
N u_{D}=0.027 \operatorname{Re}_{D}^{0.8} \operatorname{Pr}^{0.33}\left(\frac{\mu_{b}}{\mu_{w}}\right)^{0.14}
$$


Donde $\mu_{\mathrm{b}}$ es la viscosidad evaluada a la temperatura del fluido y $\mu_{\mathrm{w}}$ viscosidad evaluada a la temperatura de la pared.

Correlación Dittus Boelter se presenta en ecuación (10), Fuente: (Dittus et al., 1930):

$$
N u_{D}=0.023 \operatorname{Re}_{D}^{4 / 5} \operatorname{Pr}^{n}(10)
$$

Considera a $n=0.3$ cuando el fluido se enfría y $n=0.4$ cuando el fluido se calienta.

\subsubsection{Flujo Externo}

Los fenómenos de la transferencia de calor a través de cilindros y tubos están relacionados con la naturaleza del flujo. Esto es debido al comportamiento de la capa límite, motivo por el cual se han realizado estudios de la separación del flujo. Por lo tanto, se han desarrollado varias correlaciones empíricas para el coeficiente de transferencia de calor.

Correlación de Kern se presenta en ecuación (11), donde $\mathrm{G}_{\mathrm{s}}$ es el caudal másico lado carcasa, $\mathrm{D}_{\mathrm{e}}$ es un diámetro equivalente, Fuente: (Kern, 1973):

$\frac{h_{o} D_{e}}{k}=0.36\left(\frac{D_{e} G_{s}}{\mu}\right)^{0.55}\left(\frac{C p \mu}{k}\right)^{0.33}\left(\frac{\mu}{\mu_{b}}\right)^{0.14}$

Correlación de Hilpert se representa en ecuación (12), Fuente: (Hilpert, 1933):

$N u_{D}=C \operatorname{Re}_{D}^{m} \operatorname{Pr}^{0.33}$

Los coeficientes “C” y “m” se pueden estimar de acuerdo con el número de Reynolds:

\begin{tabular}{|crr}
\hline $\mathbf{R e}_{\mathbf{D}}$ & $\mathbf{C}$ & $\mathbf{~ m}$ \\
\hline $0.4-4$ & 0.989 & 0.330 \\
$4-40$ & 0.911 & 0.385 \\
$40-4000$ & 0.683 & 0.466 \\
$4000-40000$ & 0.193 & 0.618 \\
$40000-400000$ & 0.027 & 0.805 \\
\hline
\end{tabular}

Correlación de Zukauskas se presenta en ecuación (13), Fuente:(Zukauskas, 1987):

\begin{tabular}{cll}
$N u_{D}=C R e_{D, \max }^{m} \operatorname{Pr}^{0.36}\left(\frac{P r}{P r_{S}}\right)^{0.25}$ & & \multicolumn{1}{c}{ } \\
& \multicolumn{1}{c}{$\mathbf{C}$} & \multicolumn{1}{c}{$\mathbf{~}$} \\
\hline $10 \quad<\operatorname{Re} \leq 100$ & 0.9 & 0.4 \\
$100<\operatorname{Re} \leq 1000$ & 0.683 & 0.466 \\
$1000<\operatorname{Re} \leq 2 \mathrm{E}^{+05}$ & 0.35 & 0.6
\end{tabular}

Donde Pr es el Prandtl medido a la temperatura del fluido y $\operatorname{Pr}_{\mathrm{s}}$ es el Prandtl medido a la temperatura de superficie.

Correlación de Churchill y Bernstein se presenta en ecuación (14), Fuente: (Churchill et al., 1975; Churchill et al., 1977):

$$
N u_{D}=0.3+\frac{0.62 \operatorname{Re}_{D}^{1 / 2}+\operatorname{Pr}^{1 / 3}}{\left[1+\left(\frac{0.4}{P r}\right)^{2 / 3}\right]^{1 / 4}}\left[1+\left(\frac{R e_{D}}{282000}\right)^{5 / 8}\right]^{4 / 5}
$$

La Figura 6 muestra una ventana gráfica del programa de simulación realizados en el software EES (Engineering Equation Solver), se puede apreciar que existen espacios donde se introduce la geometría y el arreglo de los tubos del intercambiador de calor es triangular; así como las condiciones de operación de entrada de los fluidos.

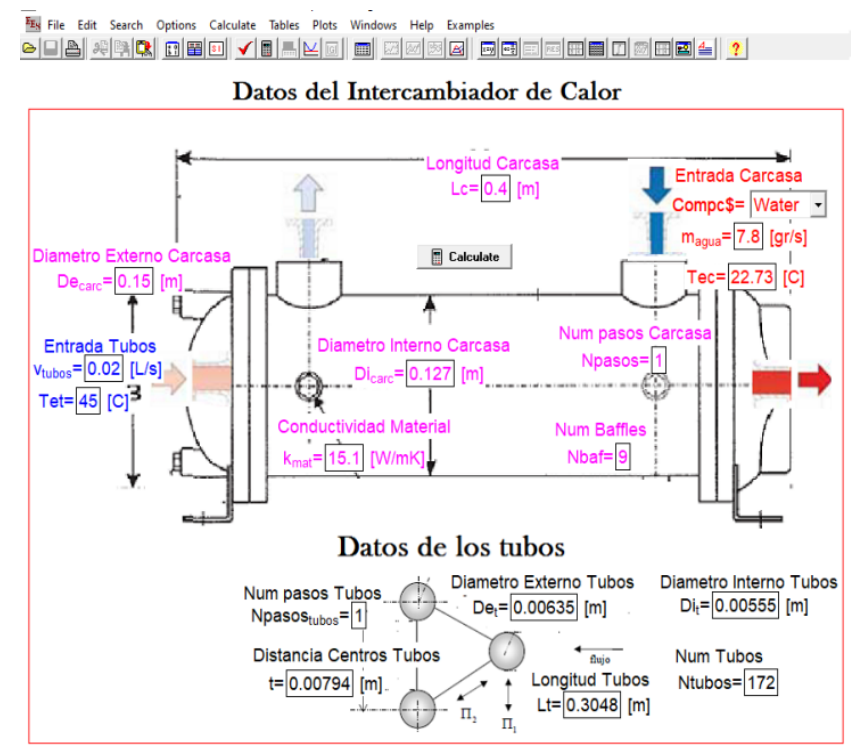

Figura 6: Datos de entrada del modelo de simulación del intercambiador de calor con arreglo triangular.

Los resultados obtenidos son las temperaturas de salida del aceite y del agua, coeficiente global de transferencia de calor, coeficientes convectivos para flujo interno y externo con las correlaciones mencionadas anteriormente, el área total de transferencia de calor, eficacia, calor total y las caídas de presión son mostrados en la Figura 7 con el software EES.

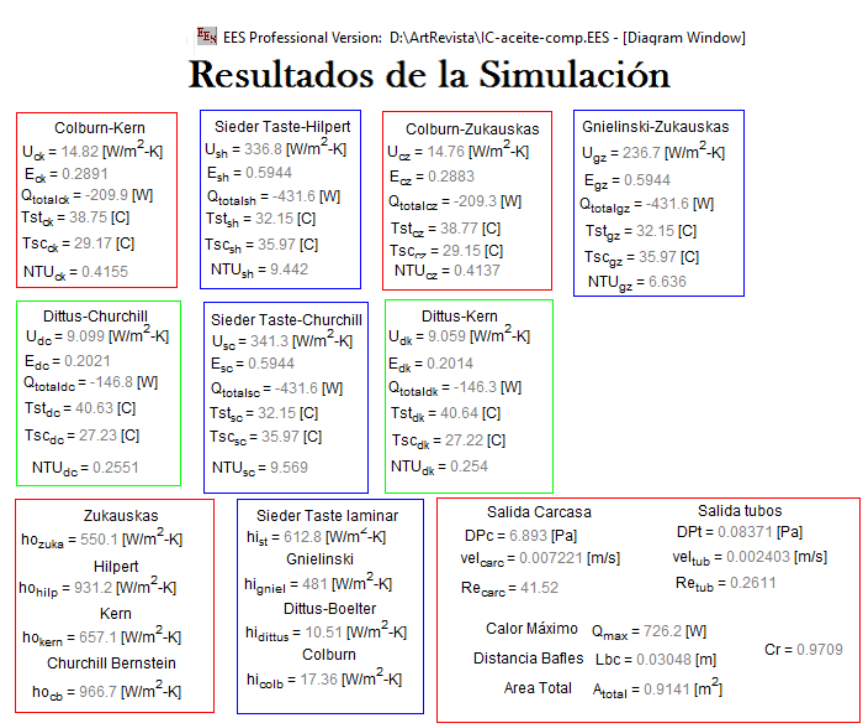

Figura 7: Resultados de la simulación con EES.

\section{Resultados}

La Figura 8 Muestra los resultados de los coeficientes convectivos internos para las diferentes pruebas que se realizaron en el enfriador de aceite. Se observa que los resultados obtenidos con Colburn y Dittus Boelter son muy bajos y cercanos entre sí, mientras los resultados de Gnielinski y Sieder Taste $N u_{D}$, presentan valores altos para todas las pruebas, donde los coeficientes convectivos internos superan las mil unidades. 


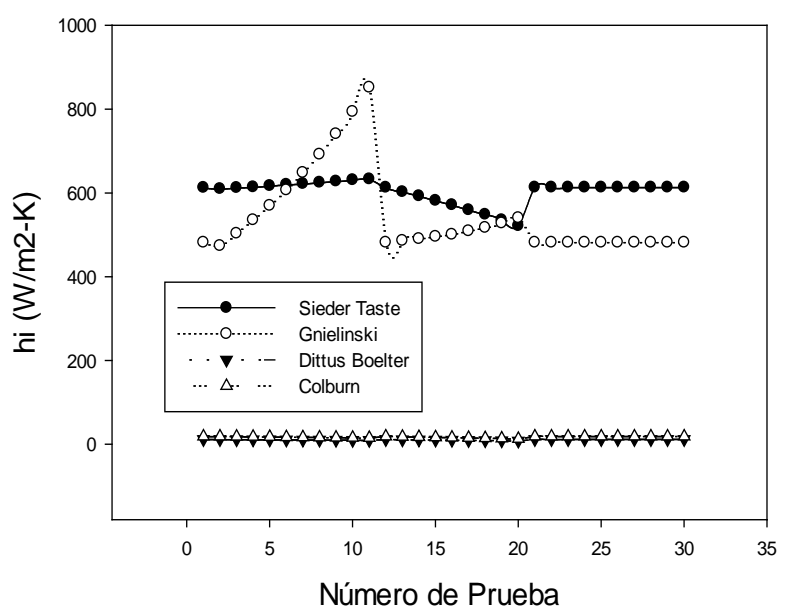

Figura 8: Resultados de los coeficientes convectivos internos.

Los resultados de los coeficientes convectivos externos son mostrados en la Figura 9. Se observa que el coeficiente convectivo más alto para todas las pruebas fue el de Churchill, mientras los resultados obtenidos con la correlación de Zukauskas fueron más bajos.

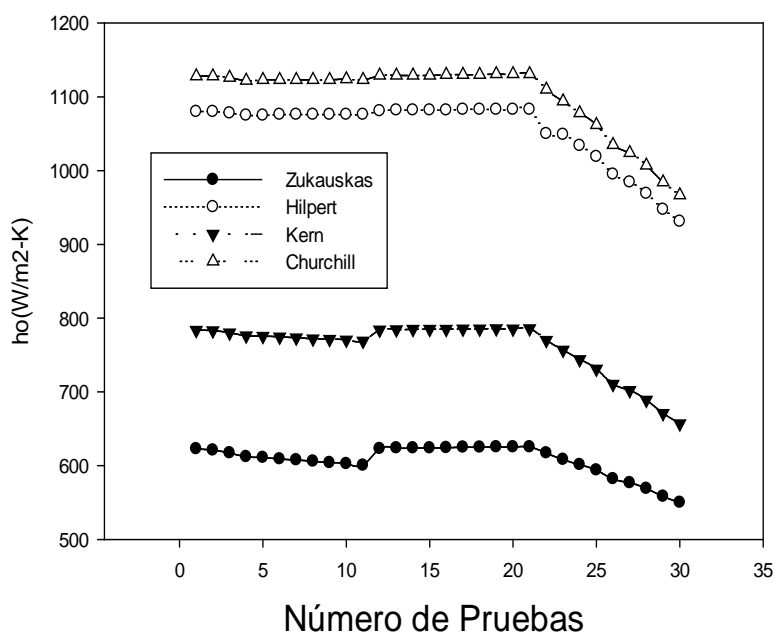

Figura 9. Resultados de los coeficientes convectivos externos.

La Figura 10 muestra los resultados de los coeficientes globales de transferencia de calor obtenidos con las diferentes combinaciones de correlaciones para flujo interno y externo. Los resultados son: Dittus Boelter-Churchill, Colburn-Zukauskas, Dittus Boelter-Kern y Colburn-Kern en un rango de 0 a $50 \mathrm{~W} / \mathrm{m}^{2}$ K. En cambio, los resultados de las demás combinaciones Sieder Taste-Hilpert, Gnielinski-Zukauskas y Sieder Taste-Churchill están en el rango de 240 a $380 \mathrm{~W} / \mathrm{m}^{2}-\mathrm{K}$.

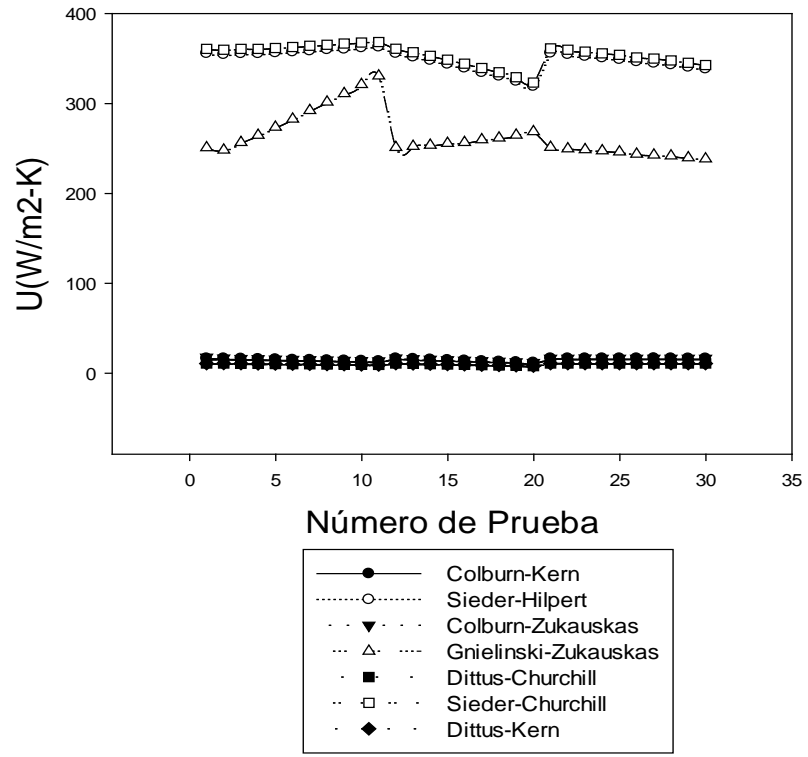

Figura 10: Resultados de los coeficientes globales de transferencia de calor con las diferentes combinaciones de correlaciones.

Los resultados de la transferencia de calor para las diferentes pruebas son mostrados en la Figura 11. Las combinaciones con Colburn-Kern, Colburn-Zukauskas, Dittus Boelter-Churchill y Dittus Boelter-Kern son las que se aproximan entre sí y están en el rango de 80 a $240 \mathrm{~W}$, mientras que las demás combinaciones están en el rango de 300 a 530 W.

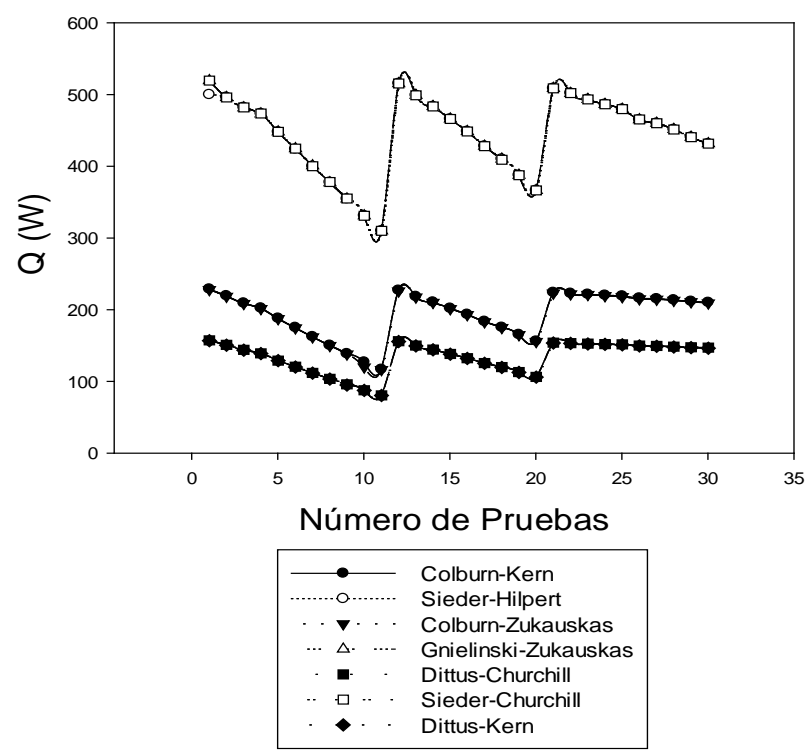

Figura 11: Resultados de la transferencia de calor con las diferentes combinaciones de correlaciones.

Las Tablas 2, 3, 4 y 5 muestran los resultados de los 30 casos de estudio que se realizaron en el enfriador de aceite. Se realiza un comparativo de las temperaturas de salida del lado del aceite y del agua obtenidas experimentalmente y con las combinaciones de correlaciones. Los resultados de las temperaturas de salida obtenidas con las correlaciones Sieder Taste-Hilpert, GnielinskiZukauskas y Sieder Taste-Churchill son las que se aproximan a los resultados experimentales en comparación de las combinaciones Colburn-Kern, Colburn-Zukauskas, DittusBoelter-Churchill y Dittus Boelter-Kern. 
Tabla 2: Comparacion de resultados de las temperaturas de salida del aceite y agua.

\begin{tabular}{|c|c|c|c|c|}
\hline \multirow[b]{2}{*}{ Prueba } & \multicolumn{2}{|c|}{ Experimental } & \multicolumn{2}{|c|}{ Colburn-Kern } \\
\hline & $\begin{array}{c}\text { Temp. } \\
\text { Salida } \\
\text { Aceite } \\
\left({ }^{\circ} \mathrm{C}\right)\end{array}$ & $\begin{array}{c}\text { Temp. } \\
\text { Salida } \\
\text { Agua } \\
\left({ }^{\circ} \mathrm{C}\right)\end{array}$ & $\begin{array}{c}\text { Temp. } \\
\text { Salida } \\
\text { Aceite } \\
\left({ }^{\circ} \mathrm{C}\right)\end{array}$ & $\begin{array}{c}\text { Temp. } \\
\text { Salida } \\
\text { Agua } \\
\left({ }^{\circ} \mathrm{C}\right)\end{array}$ \\
\hline 1 & 34.08 & 31.1 & 38.19 & 26.95 \\
\hline 2 & 32.42 & 30.5 & 37.46 & 26.77 \\
\hline 3 & 32.26 & 31.5 & 36.75 & 26.17 \\
\hline 4 & 31.04 & 29.32 & 35.96 & 25.37 \\
\hline 5 & 30.07 & 29.1 & 35.38 & 25.18 \\
\hline 6 & 30.58 & 29.37 & 34.76 & 24.92 \\
\hline 7 & 29.9 & 28.24 & 34.13 & 24.7 \\
\hline 8 & 30 & 28.51 & 33.5 & 24.44 \\
\hline 9 & 29.03 & 28 & 32.84 & 24.2 \\
\hline 10 & 27.09 & 27.6 & 32.18 & 23.98 \\
\hline 11 & 27.65 & 26.5 & 31.48 & 23.7 \\
\hline 12 & 34.19 & 32.36 & 38.24 & 27.08 \\
\hline 13 & 35.07 & 32.59 & 38.16 & 26.92 \\
\hline 14 & 34.02 & 32.83 & 38.08 & 26.78 \\
\hline 15 & 33.22 & 33 & 37.97 & 26.59 \\
\hline 16 & 33.48 & 33.3 & 37.89 & 26.45 \\
\hline 17 & 33.08 & 33 & 37.8 & 26.34 \\
\hline 18 & 33 & 29.9 & 37.7 & 26.15 \\
\hline 19 & 32.78 & 29.5 & 37.6 & 26.02 \\
\hline 20 & 32.6 & 31.2 & 37.45 & 25.79 \\
\hline 21 & 34.02 & 31.5 & 38.33 & 27.33 \\
\hline 22 & 34.03 & 31.5 & 38.37 & 27.48 \\
\hline 23 & 34.35 & 31.8 & 38.41 & 27.67 \\
\hline 24 & 34.46 & 32 & 38.44 & 27.83 \\
\hline 25 & 34.56 & 32 & 38.48 & 28 \\
\hline 26 & 34.82 & 32.1 & 38.57 & 28.35 \\
\hline 27 & 34.94 & 32.3 & 38.59 & 28.47 \\
\hline 28 & 35.14 & 32.7 & 38.65 & 28.69 \\
\hline 29 & 35.32 & 33 & 38.7 & 28.95 \\
\hline 30 & 35.42 & 33.6 & 38.75 & 29.17 \\
\hline
\end{tabular}

Tabla 3: Comparación de resultados de las temperaturas de salida del aceite teóricas y agua.

\begin{tabular}{|c|c|c|c|}
\hline \multicolumn{2}{|c|}{ Sieder Taste-Hilpert } & \multicolumn{2}{|c|}{ Colburn-Zukauskas } \\
\hline $\begin{array}{l}\text { Temp. } \\
\text { Salida }\end{array}$ & $\begin{array}{l}\text { Temp. } \\
\text { Salida }\end{array}$ & $\begin{array}{l}\text { Temp. } \\
\text { Salida }\end{array}$ & $\begin{array}{l}\text { Temp. } \\
\text { Salida }\end{array}$ \\
\hline Aceite & Agua & Aceite & Agua \\
\hline$\left({ }^{\circ} \mathrm{C}\right)$ & $\left({ }^{\circ} \mathrm{C}\right)$ & $\left({ }^{\circ} \mathrm{C}\right)$ & $\left({ }^{\circ} \mathrm{C}\right)$ \\
\hline 33.37 & 33.37 & 38.22 & 26.93 \\
\hline 32.88 & 32.88 & 37.48 & 26.75 \\
\hline 32.19 & 32.19 & 36.77 & 26.16 \\
\hline 31.36 & 31.36 & 35.98 & 25.35 \\
\hline 30.92 & 30.92 & 35.4 & 25.17 \\
\hline
\end{tabular}

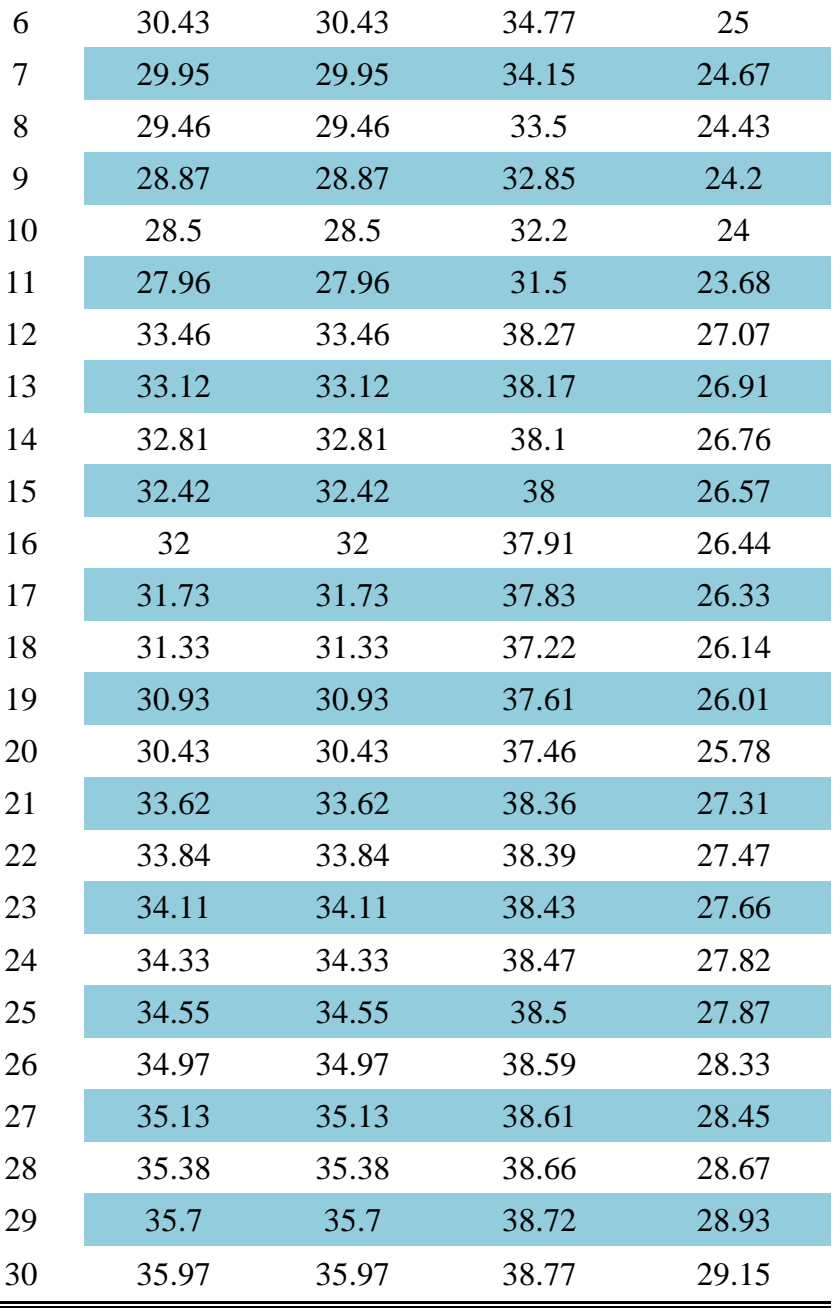

Tabla 4: Comparación de resultados de las temperaturas de salida del aceite y agua.

\begin{tabular}{|c|c|c|c|}
\hline \multicolumn{2}{|c|}{ Gnielinski-Zukauskas } & \multicolumn{2}{|c|}{ Dittus-Churchill } \\
\hline $\begin{array}{l}\text { Temp. } \\
\text { Salida }\end{array}$ & $\begin{array}{l}\text { Temp. } \\
\text { Salida }\end{array}$ & $\begin{array}{l}\text { Temp. } \\
\text { Salida }\end{array}$ & $\begin{array}{l}\text { Temp. } \\
\text { Salida }\end{array}$ \\
\hline Aceite & Agua & Aceite & Agua \\
\hline$\left({ }^{\circ} \mathrm{C}\right)$ & $\left({ }^{\circ} \mathrm{C}\right)$ & $\left({ }^{\circ} \mathrm{C}\right)$ & $\left({ }^{\circ} \mathrm{C}\right)$ \\
\hline 29.54 & 33.3 & 40.32 & 25.37 \\
\hline 29.21 & 32.8 & 39.5 & 25.25 \\
\hline 28.63 & 32.2 & 38.7 & 24.73 \\
\hline 27.85 & 31.4 & 37.84 & 23.97 \\
\hline 27.61 & 30.9 & 37.13 & 23.9 \\
\hline 27.28 & 30.3 & 36.4 & 23.71 \\
\hline 26.98 & 29.99 & 35.65 & 23.57 \\
\hline 26.66 & 29.5 & 34.89 & 23.41 \\
\hline 26.34 & 28.99 & 34.13 & 23.25 \\
\hline 26 & 28.7 & 33.36 & 23.12 \\
\hline 25.66 & 27.99 & 32.57 & 22.9 \\
\hline 29.66 & 33.47 & 40.36 & 25.53 \\
\hline 29.36 & 33.23 & 40.3 & 25.41 \\
\hline 29.1 & 32.88 & 40.25 & 25.35 \\
\hline 28.77 & 32.45 & 40.19 & 25.18 \\
\hline 28.51 & 32.1 & 40.14 & 25.1 \\
\hline
\end{tabular}




\begin{tabular}{l|cccc}
17 & 28.24 & 31.78 & 40 & 25 \\
18 & 27.93 & 31.36 & 40 & 24.92 \\
19 & 27.65 & 30.99 & 39.95 & 24.86 \\
20 & 27.26 & 30.49 & 39.86 & 24.69 \\
21 & 29.86 & 33.68 & 40.42 & 25.78 \\
22 & 30.06 & 33.87 & 40.43 & 25.9 \\
23 & 30.31 & 34.18 & 40.45 & 26.05 \\
24 & 30.52 & 34.37 & 40.47 & 26.17 \\
25 & 30.72 & 34.7 & 40.5 & 26.3 \\
26 & 31.15 & 34.7 & 40.54 & 26.6 \\
27 & 31.3 & 35.3 & 40.55 & 26.68 \\
28 & 31.56 & 35.5 & 40.58 & 26.86 \\
29 & 31.88 & 35.9 & 40.61 & 27.06 \\
30 & 32.15 & 35.8 & 40.63 & 27.23 \\
\hline \hline
\end{tabular}

Tabla 5: Comparación de resultados de las temperaturas de salida del aceite teóricas y agua.

\begin{tabular}{cccc}
\hline \multicolumn{2}{c}{ Sieder-Churchill } & \multicolumn{2}{c}{ Dittus-Kern } \\
Temp. & Temp. & Temp. & Temp. \\
Salida & Salida & Salida & Salida \\
Aceite & Agua & Aceite & Agua
\end{tabular}

\begin{tabular}{ccccc} 
Prueba & $\mathbf{( { } ^ { \circ } \mathbf { C } )}$ & $\mathbf{( { } ^ { \mathbf { } } \mathbf { C } )}$ & $\mathbf{( { } ^ { \circ } \mathbf { C } )}$ & $\mathbf{(} \mathbf{C})$ \\
\hline \hline 1 & 29.53 & 33.1 & 40.33 & 25.36 \\
2 & 29.21 & 32.7 & 39.52 & 25.24 \\
3 & 28.62 & 32.3 & 38.72 & 24.72 \\
4 & 27.85 & 31.6 & 37.86 & 23.96 \\
5 & 27.6 & 30.7 & 37.14 & 23.88 \\
6 & 27.28 & 30.5 & 36.4 & 23.71 \\
7 & 26.98 & 29.8 & 35.66 & 23.56 \\
8 & 26.66 & 29.6 & 35 & 23.4 \\
9 & 26.34 & 28.8 & 34.14 & 23.24 \\
10 & 26.04 & 28.7 & 33.37 & 23.11 \\
11 & 25.66 & 27.8 & 32.58 & 22.9 \\
12 & 29.65 & 33.7 & 40.37 & 25.52 \\
13 & 29.36 & 33.3 & 40.31 & 25.4 \\
14 & 29.1 & 32.9 & 40.26 & 25.31 \\
15 & 28.77 & 32.6 & 40.2 & 25.18 \\
16 & 28.51 & 32.1 & 40.16 & 25.1 \\
17 & 28.24 & 31.8 & 40.1 & 25.05 \\
18 & 27.93 & 31.4 & 40 & 24.92 \\
19 & 27.65 & 31 & 39.96 & 24.86 \\
20 & 27.26 & 30.4 & 39.87 & 24.68 \\
21 & 29.85 & 33.5 & 40.43 & 25.77 \\
22 & 30.06 & 33.9 & 40.45 & 25.87 \\
23 & 30.31 & 34.2 & 40.47 & 26.07 \\
24 & 30.52 & 34.4 & 40.49 & 26.16 \\
25 & 30.72 & 34.58 & 40.5 & 26.27 \\
26 & 31.15 & 34.6 & 40.55 & 26.55 \\
27 & 31.3 & 35.4 & 40.57 & 26.67 \\
& & & & \\
\hline 1
\end{tabular}

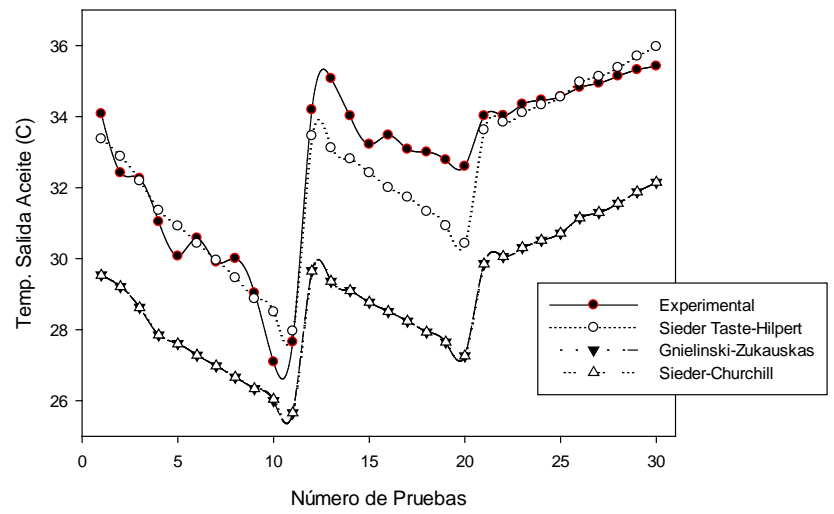

Figura 12: Resultados de temperaturas de salida lado tubos (aceite) con las 3 combinaciones de correlaciones aproximadas a las experimentales.

Los resultados de las temperaturas de salida del agua lado carcasa son mostrados en la Figura 13, para este caso los datos obtenidos con las 3 combinaciones de correlaciones (Sieder Taste-Hilpert, Gnielinski-Zukauskas y Sieder Taste-Churchill) son muy semejantes entre ellos y se aproximan a los datos experimentales.

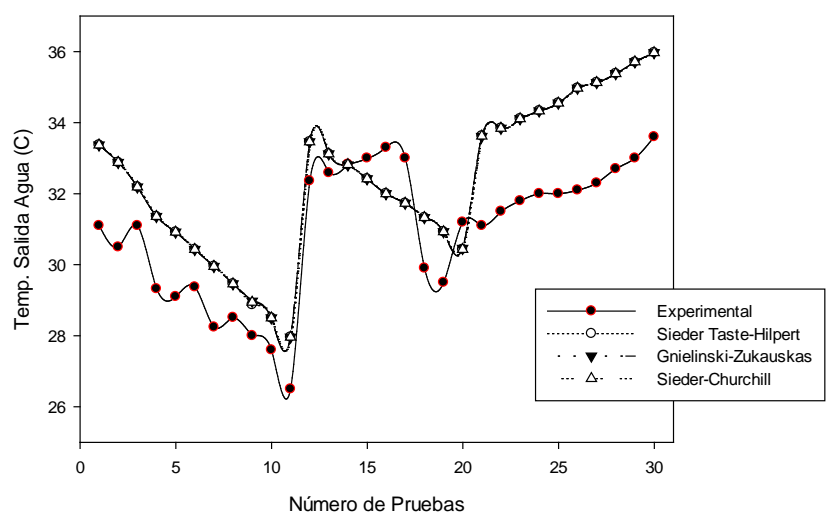

Figura 13: Resultados de temperaturas de salida lado carcasa (agua) con las 3 combinaciones de correlaciones aproximadas a las experimentales.

\section{Conclusiones}

Las combinaciones de correlaciones Sieder Taste-Hilpert se aproximaron a los resultados experimentales de las temperaturas de salida del aceite y agua, se ajustaron al modelo experimental del enfriador de aceite para los 30 casos de estudio. Los datos obtenidos con las combinaciones Gnielinski-Zukauskas y Sieder Taste-Churchill se aproximaron a los resultados experimentales del lado carcasa (agua) pero del lado tubos (aceite) los datos no fueron muy aproximados. En cambio, los resultados con ColburnKern, Colburn-Zukauskas, Dittus Boelter-Churchill y Dittus- 
Boelter-Kern no se adaptaron al modelo del enfriador ya que las correlaciones presentan restricciones para su empleo en los equipos térmicos, por lo tanto, no todas las correlaciones encontradas en la literatura se ajustan a los intercambiadores de calor porque dependen de los números de Reynolds, Prantl, diseño del intercambiador de calor, etc. La revisión bibliográfica demostró que no hay una metodología para predecir el rendimiento del equipo. Por esta razón, en este trabajo, se desarrolló una metodología para diseñar y predecir el rendimiento, en miras de optimizar la eficiencia energética, seguridad y facilidad de servicio de los intercambiadores de calor tipo coraza y tubos.

\section{Conflicto de intereses}

Los autores declaran que no existen conflictos de intereses.

\section{Referencias}

Kakac, S., Liu, H., (1998). Heat Exchanger Selection, Rating and Thermal Design. by CRC Press LLC. ISBN 9781466556164

Incropera, F. P., DeWitt, D. P., (1999) Fundamentos de Transferencia de Calor. Cuarta Edición. Ed. Prentice Hall, México. ISBN 9781119582786 1119582784

Gaddis, D. (Editor2007). Standard of the Tubular Exchanger Manufacturers Association. Tarrytown (NY): TEMA Inc.

Kern, D. Q., (1973). Process Heat Transfer. New York. McGraw-Hill.

Bell, K. J., (1981). Delaware method for shell side design. In: Kakac S, Bergles AE, Mayinger F, Editors. Heat Exchanger: Thermalhydraulicfundamentals and design. New York. p. 581-618.ISBN: 0-82479787-6.

Gay, B., Jenkins, J. D.; Mackley, N. V., (1982). Shell-side heat transfer in double-segmentally baffled cylindrical shell-and-tube exchangers. Int. J. Heat Mass Transfer. 9(1), 39-47. DOI: 10.1016/0094-4548(82)90046-7

Gaddis, E. S., Gnielinski, V., (1997). Pressure drops on the shell side of shelland-tube heat exchangers with segmental baffles. Chem. Eng. Process. 36,149-59.

Karno, A., Ajib, S., (2006). Effect of tube pitch on heat transfer in shell-andtube heat exchangers-new simulation software. Int. J. Heat Mass Transfer 42, 263-70. DOI: 10.1007/s00231-005-0002-9
Eryener, D., (2006). Thermoeconomic optimization of baffle spacing for shell and tube heat exchangers. Energy Convers Manage. 47, 1478-89. DOI: 10.1016/j.enconman.2005.08.001

Keene, L.W., (1994). Computer Modeling Validation for Shell and Tube Heat Exchangers. Institution of Chemical Engineers, England, pp. 611-615. DOI: $10.1615 /$ IHTC10.5410

Reppich, M., Zagermann, S., (1995). New design method for segmentally baffled heat exchanger. Compute. Chem. Eng. 19. pp. 137-142. DOI: 10.1016/0098-1354(95)87028-8

Leong, K. C., Toh, K. C., Leong, Y. C., (1998). Shell and Tube Heat Exchanger Design Software for Educational Applications. International Journal of Engineering Education. 14, (3), 217-224.

Thet, S. M. T., Aung, K. L., Sandar, M. M., (2008). Heat Exchanger Design. World Academy of Science. Engineering and Technology 46. DOI: 10.5281/zenodo.1334209

El-Fawal, M. M., Fahmy, A. A., B. M. Taher.,(2011). Modelling of Economical Design of Shell and Tube Type Heat Exchanger Using Specified Pressure Drop. Journal of American Science. 7, (12) 32-40. ISSN: 1545-1003.

Kara, Y. A., Guraras, O., (2004). A computer program for designing of shell and tube heat exchanger. Applied Thermal Engineering 24, pp. $1797-$ 1805. DOI: 10.1016/j.applthermaleng.2003.12.014.

Fettaka, S., Thibault, J., Gupta, Y., (2013). Design of shell and tube heat exchanger using multiobjetive optimization. Int. J. Heat Mass Transfer 60, pp. 343-354. DOI: 10.1016/j.ijheatmasstransfer.2012.12.047.

Colburn, A. P., (1933). A method for correlating forced convection heat transfer data and a comparison with fluid friction. Trans. Am. Inst. Chem. Eng, 29, 174.

Gnielinski, V., (1976). New equation for heat and mass transfer in turbulent pipe and channel flow. Int. Chem. Eng. 16, 359.

Sieder, E. N., G. E., Taste., (1936). Heat Transfer and Pressure Drop of Liquid in Tubes. Ind. Eng. Chem. 28 (12), pp. 1429-1435. DOI: 10.1021/ie50324a027

Dittus, F. W, L. M. K. Boelter., (1930). University of California Berkeley, Publications on Engineering, 2, pp. 443.

Hilpert, R., (1933). Wärmeabgue von Geheizten Drähten und Rohrenim Lufstrom. Forsch. Geb. Ingenieurwes. 4, 215.

Zukauskas, A. A., (1987). Convective heat transfer in cross flow. In handbook of single-phase convective heat transfer. Kakac S, Shah R. K, and Aung W. Eds John Wiley and Sons, New York. ISBN 0-471-81702-3

Churchill, S. W., Chu, H. H. S., (1975). Correlating Equations for Laminar and Turbulent Free Convection from a Horizontal Cylinder. Int. J. Heat Mass Transfer. 18, pp. 1049-1053. DOI: 10.1016/0017-9310(75)90222-7

Churchill, S. W., M. Bernstein., (1977). A correlating Equation for Forced Convection from Gases and Liquids to a Circular Cylinder in Crossflow, J. Heat Mass Transfer, 99, pp. 300-306.DOI: 10.1115/1.3450685 